\title{
Clinical Significance of Circulating Serum Levels of SCD95 and TNF- $\alpha$ in Cytoprotection of Cervical Cancer
}

\author{
Saurabh Kumar Agnihotri ${ }^{1,2 \#}$, Balawant Kumar" ${ }^{3 \#}$, Ankita Jain², Anjali Anjali ${ }^{3,4}$, \\ Mahendra Pal Singh Negi ${ }^{3}$, Rekha Sachan ${ }^{4}$, Madan Lal Brahma Bhatt ${ }^{1}$, \\ Raj Kamal Tripathi ${ }^{3,4}$, Monika Sachdev*2,5
}

\begin{abstract}
Background: This study correlates the serum levels of sCD95 \& TNF- $\alpha$ with a simple cell-based assay to evaluate the capacity of the serum sample to induce apoptosis in Jurkat cells. Interlinking of these parameters can be explored to design a minimum invasive diagnostic strategy for cervical cancer (CC).

Methods: Sera samples were assessed to induce apoptosis in Jurkat cells through FACS. Serum levels of SCD95 and TNF- $\alpha$ were measured by ELISA. JNK phosphorylation was evaluated in sera incubated Jurkat cells. Data was scrutinized through statistical analysis.

Results: Significantly higher serum levels of sCD95 and lower TNF- $\alpha$ levels were observed in CC patients; their sera samples inhibited induction of apoptosis in Jurkat cells through reduced JNK phosphorylation. Statistical analysis linked these three parameters for the early screening of CC.

Conclusions: Distinct sera levels of sCD95 \& TNF- $\alpha$ in CC patients showed an anti-apoptotic effect, which can be considered for early detection of CC.
\end{abstract}

Keywords: Apoptosis, sCD95, Jurkat Cells, Tumor Necrosis Factor-alpha, Uterine Cervical Neoplasms.

\section{Introduction}

Apoptotic pathways are usually disrupted or inactivated in cancerous cells and engenders uncontrolled cell growth and tumor cell resistance (1). Apart from internal cytotoxic stress that predominantly triggers apoptosis, it can also be induced by cell-membraneanchored signalling pathways of the TNFsuperfamily: the CD95-receptor/CD95-ligandsystem (Fas/FasL or APO-1) and the tumor necrosis factor (TNF)-related apoptosis inducing ligand (TRAIL or APO-2L) with the TRAIL receptors 1 and 2 (TRAIL-R1 and R2) $(2,3)$. The best known death receptor is APO1/ Fas, which induces apoptosis through recruiting initiator caspases into the deathinducing signalling complex (DISC) which leads to their activation (4). CD95 is also known to activate non-apoptotic pathways including $\mathrm{NF} \kappa \mathrm{B}, \quad$ Erk1/2\& JNK1/2, p38 mitogen-activated protein kinase pathways and the 3-kinase/Akt phosphatidylinositol pathway $(5,6)$.

CD95 consists of two isoforms, one is a soluble form (sCD95) and other one is anchored to the cellular membrane (mCD95) $(7,8)$. Binding of sCD95 to CD95L leads to unavailability of CD95L to bind with mCD95 and leads to the downregulation of cellular 
apoptosis. Elevated levels of sCD95 have been reported in sera samples of patients with various malignancies (9-12). Soluble form of CD95L is generated by a metalloproteinaselike protease, which binds to sCD95 and induce apoptosis in cytotoxic T-cells thus preventing the recognition of tumor cells (13). Therefore, detection of sCD95 in serum could be a new approach to detect the cancer.

Basically, CD95 is a membrane protein belonging to the TNF family $(14,15)$. TNF- $\alpha$ is a pleiotropic inflammatory cytokine, critical for the various cellular events. TNF- $\alpha$ may exist as a $26-\mathrm{kDa}$ membrane tethered form (mTNF- $\alpha$ ) or a soluble $17-\mathrm{kDa}$ cytokine (sTNF- $\alpha$ ). Both these isoforms have contradictory effects on tumor growth but the mechanism has not been understood properly (16).

Usually CC remains asymptomatic and goes undiagnosed, but can be cured if detected early. Presently Pap test is widely used to detect precancerous changes in the cervix, which may lead to the progression of CC (17) but has low sensitivity $(18,19)$. Numerus aspects limits the test sensitivity including lesion size, inaccessible location, lesser number of abnormal cells as well as their small size; manifestation of blood due to inflammation further complicates the cell visualization. The persistent infection of human papillomavirus (HPV) also leads to high-grade cervical intraepithelial neoplasia (CIN) $(20,21)$ and ultimately progress to CC (22). Most of the clinically confirmed CC cases are associated with infection of high-risk HPV $(16,20,24)$ and can be transmitted through sexual activity $(25,26)$. Consequently, the combination of the Pap test with HPVDNA testing can increase noteworthy sensitivity, but methodological and economic constrains limits its feasibility.

Therefore, it is warranted to look for novel simple and cost-effective complementary prognostic methods for accurate and early prediction of the disease. In the present study, comparatively, a simpler and more authentic approach has been established to predict the risk of developing $\mathrm{CC}$ based on three different parameters of patients' sera samples. These parameters include the sera levels of sCD95 and TNF- $\alpha$ as well as capacity to induce apoptosis in Jurkat $\mathrm{T}$ cells through the patients' serum factors.

\section{Materials and Methods Ethics statement}

The study was conducted according to the guidelines of the declaration of Helsinki and approved by the Institutional Ethics Committee (Approval ref\# 51 E.C.M. IIA/P1), Office of the Research Cell, King George's Medical University, Lucknow. Written informed consents were obtained from all enrolled participants to participate in this study.

Collection of blood samples and separation of sera Blood samples $(\sim 5 \mathrm{ml})$ were collected from the CC patients $(n=20)$ visiting King George's Medical University Lucknow, along with age matched $(n=20)$ healthy controls (HC) by venipuncture in serum separating vacutainers (367956, BD). After blood clotting, serum was separated through centrifugation in $2000 \mathrm{~g}$ for 10 minutes at $4{ }^{\circ} \mathrm{C}$ and stored at $-80{ }^{\circ} \mathrm{C}$ until further analysis.

\section{Cell lines and cell culture}

Human T lymphocyte cell line Jurkat cells and human embryonic kidney cell line HEK-293 were obtained from institutional cell repository, originally procured from ATCC (American Type Cell Culture). Jurkat \& HEK293 cells were maintained in RPMI and DMEM high glucose media respectively supplemented with 10\% FBS (10082147, Gibco) along with $1 \mathrm{X}$ antibiotic solution (15140122, Gibco) containing $1 \mathrm{mM}$ sodium pyruvate (58636, Sigma Aldrich). Culture was maintained at $37{ }^{\circ} \mathrm{C}$ temperature and $5 \% \mathrm{CO}_{2}$ along with $\sim 90 \%$ humidity and cells were allowed for growth and proliferation.

\section{Analysis of induced apoptosis in sera incubated cells} Jurkat cells and HEK-293 cells were seeded at a density of $2.5 \times 10^{5}$ and $3 \times 10^{5}$ cells $/ \mathrm{ml}$ media respectively in each well of 6-well plates. Further, $100 \mu 1$ of serum from clinical subjects was added in the wells containing $2 \mathrm{ml}$ to total 
media. After 48 hours of incubation, apoptosis was assessed using Annexin V FITC and PI apoptosis detection kit (V13242, Invitrogen) following manufacturer's instructions. Total 10,000 events were analyzed for each sample in BD FACS Calibur flow cytometer system (USA). Cells having positive scoring for Annexin-V but negative for PI were considered as apoptotic and the subsequent data was analyzed using Cell QuesecPro software.

Quantitation of SCD95 and SCD95L in sera samples by Enzyme Linked Immunosorbent Assay (ELISA)

sCD95 and sCD95L level were measured in each serum sample with the Human APO1/FAS ELISA Kit (KHS9502, BioSource International) and Human sFAS Ligand ELISA Kit (KHS9521, BioSource International) respectively, as described by the manufacturers. The range of the kit for sCD95 was 0.23-15 $\mathrm{ng} / \mathrm{ml}$.

\section{Detection of TNF- $\alpha$ level in sera by ELISA}

Serum concentration of TNF- $\alpha$ was determined by ELISA using Human TNF- $\alpha$ Kit (KHC3011, Invitrogen) as described by the manufacturer. Twenty CC patient and 20 healthy volunteer's serum samples were thawed completely and mixed well prior to analysis. All standards, controls and samples were analyzed in duplicate. The minimum detectable dose of TNF- $\alpha$ by this kit was $1.7 \mathrm{pg} / \mathrm{ml}$.

\section{Extraction of protein and Western Blotting}

Cells were harvested after 48 hrs of sera incubation and $100 \mu \mathrm{l}$ of RIPA protein lysis buffer (R0278, Sigma Aldrich) containing 1X protease (p8340, Sigma Aldrich) and phosphatase inhibitor (p0044, Sigma Aldrich) cocktail was added per $10^{6}$ cells. After centrifugation for $12000^{*} \mathrm{~g}$ for $10 \mathrm{~min}$ at $4{ }^{\circ} \mathrm{C}$, supernatant was aspirated, and protein was quantified by Bradford reagent (B6916, Sigma Aldrich).

Around $50 \mu \mathrm{g}$ cell lysates were loaded and resolved on $12 \%$ SDS-polyacrylamide gels followed by transfer to nitrocellulose membrane (HATF00010, Millipore, Massachusetts, USA). The membrane was blocked with $2 \%(\mathrm{w} / \mathrm{v})$ bovine serum albumin in PBST (1X PBS $+0.1 \%$ Tween 20) at $25{ }^{\circ} \mathrm{C}$ for $1 \mathrm{hr}$ followed by probing with specific primary antibody (anti-phospho-p54/46JNK or anti-p54/46JNK) for overnight (14 hrs) at $4{ }^{\circ} \mathrm{C}$. After washing membrane was incubated with the specific HRP conjugated secondary antibody for $2 \mathrm{hrs}$ at $25^{\circ} \mathrm{C}$. Protein bands were detected using Immobilon Western Chemiluminescent HRP Substrate (WBKLSO500, Millipore) and developed with chemi-documentation imaging system (Image Quant LAS 4000, GE Life Science, PA, USA).

\section{Statistical analysis}

Data were summarized as Mean \pm SE (standard error of the mean). Two independent groups of $\mathrm{HC}$ and $\mathrm{CC}$ patients were compared by Student's T-test. Pearson correlation analysis was done to assess the association between the variables i.e. serum concentration of sCD95 and TNF- $\alpha$ as well as apoptosis induction of Jurkat cells through sera samples of HC Vs. CC patients. Simple linear regression analysis was done to assess the strength of association between these variables. Diagnostic accuracy (sensitivity and specificity) of these parameters (sCD95, TNF- $\alpha$ and apoptosis induction) for the patients was done through receiver operative characteristics (ROC) curve analysis. A two-tailed $(\alpha=2)$ with $p<0.05$ was considered to be statistically significant. All analysis was performed on STATISTICA 7.1 software (StatSoft, Inc., USA).

\section{Results}

Healthy serum induces apoptosis in Jurkat cells through CD95/Fas/Apo-1 pathway

Jurkat and HEK-293 cells were incubated with the cocktail of 20 healthy women sera which induced apoptosis in Jurkat cells (Fig. 1A) but not in HEK-293 cells (Fig. 1B). Results indicated that Jurkat cells were highly sensitive to serum exposure. Increased concentration of serum exposure led to higher cell death in Jurkat cells. Whereas, HEK-293 cells remained resistant to CD95L-induced apoptosis, despite of gradual increase in serum concentration and longer time of serum exposure. The apoptotic rate induced in Jurkat 
cells was $31.5 \%, 58.3 \%$ and $84.1 \%$ at 100,150 and $200 \mu \mathrm{l}$ of serum respectively (Fig. 1C). While induced apoptosis in HEK-293 cells hardly remained $9 \%$ after the exposure to healthy serum, which was similar to control

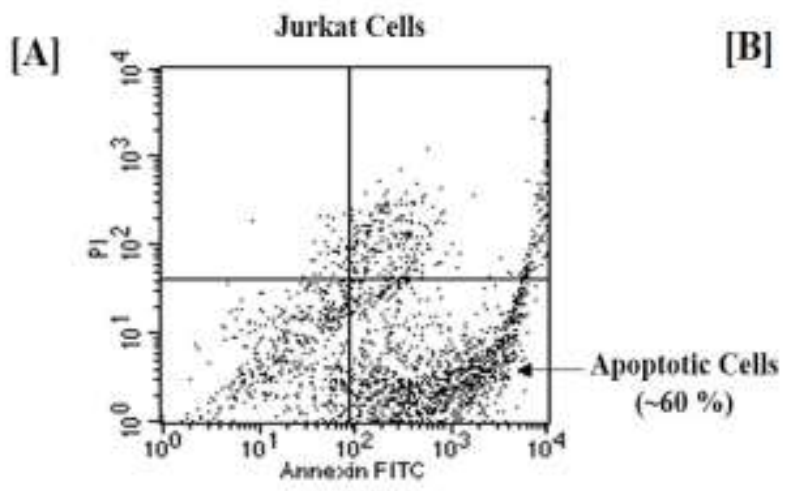

[C]

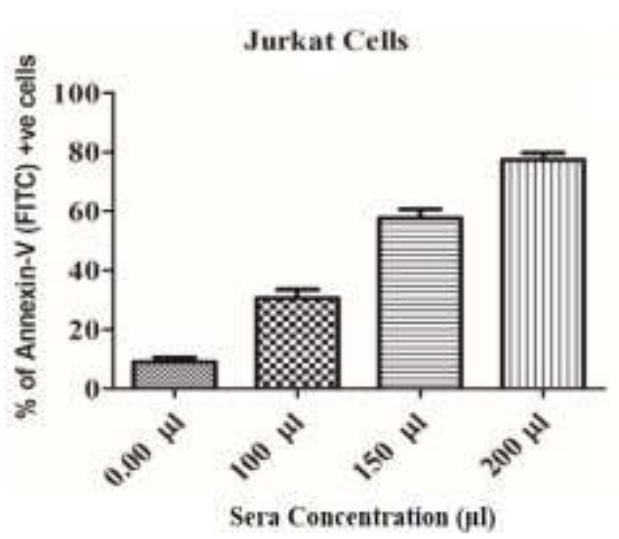

[D] cells grown without serum exposure (Fig. 1D). These observations strongly depict an association of the CD95 pathway in the apoptosis of Jurkat cells through healthy women sera.
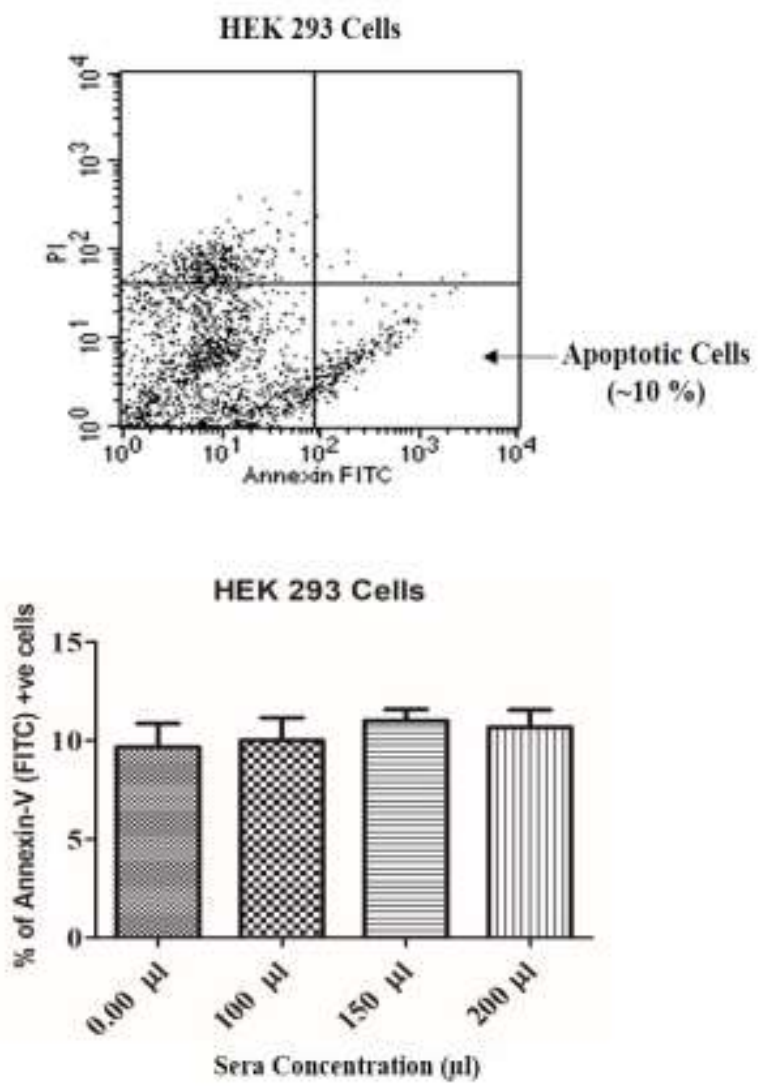

Fig. 1. Normal sera induce apoptosis in the Jurkat cells as compared to the HEK-293 cells. Representative FACS dot-plot with $150 \mu 1$ of healthy sera cocktail showing (A) Induction of apoptosis in Jurkat cells, whereas (B) HEK 293 cells did not show considerable induction of the apoptosis. (C) Jurkat cells and (D) HEK-293 cells, were incubated for 48 hrs with 0-200 $\mu \mathrm{l}$ cocktail of 20 healthy women sera. Apoptosis rate in each cell line was measured by flow cytometry using Annexin-V/PI markers. A total of 10000 events were captured by BD FACS Calibur flow cytometer system (USA) and analysis was performed with the CellQuesecPro software. The data are represented as means \pm SEM from three independent experiments.

\section{Serum induced apoptosis is mediated by JNK1/p38 phosphorylation}

It is quite evident from our results that the sera of CC subjects contain high concentration of sCD95 receptor which interact with CD95L (Fas ligand) and causes dilution of CD95L in the serum. Consequently, CD95L is unavailable in high concentration to interact with cell membrane associated CD95 receptor which could lead to activation of death receptor domain which further activates ASK1 and its downstream JNK/p38 resulting in apoptosis. Jurkat cells were incubated with sera from CC and $\mathrm{HC}$ subjects for $48 \mathrm{hrs}$ and analyzed for the level of JNK phosphorylation through western blotting (Fig. 2). This analysis indicated that Jurkat cells incubated with sera of CC subjects (Fig. 2B) showed significantly lesser activation of JNK (ratio between integrated density of phospho-p54/46JNK and Total JNK levels) as compared to the cells incubated with sera of $\mathrm{HC}$ (Fig. 2A). Further assessment for the p38 phosphorylation (data not shown) in the similar experimental set up demonstrated higher phosphorylation with the CC patients' sera, when compared with sera of healthy women. 
Overall, these results strongly suggest that apoptosis induction in Jurkat $\mathrm{T}$ cell is mediated by $\mathrm{CD} 95 / \mathrm{CD} 95 \mathrm{~L}$ interactions that activate the JNK1 and p38 downstream.

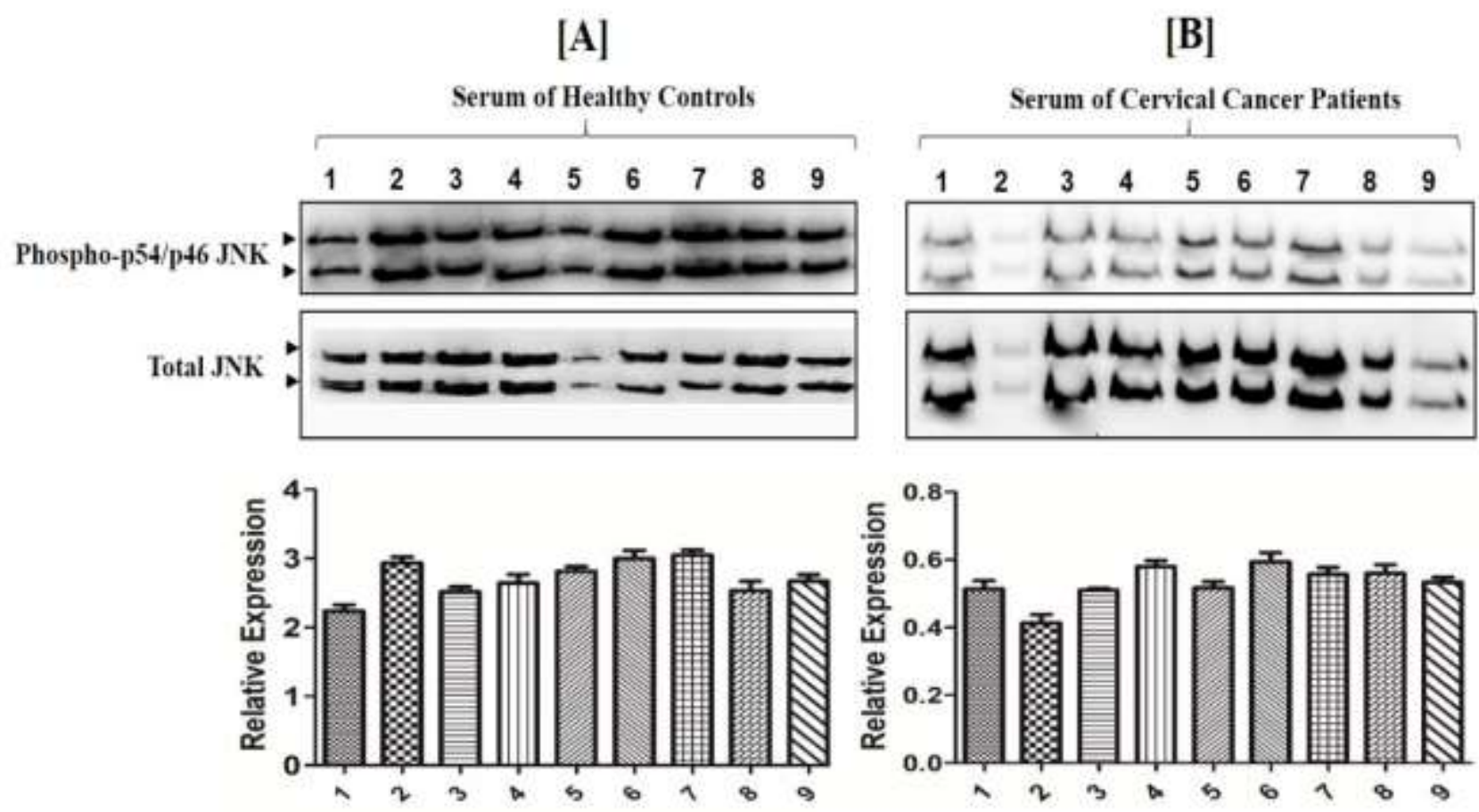

Fig. 2. Reduced JNK phosphorylation was observed during induced Apoptosis of Jurkat cells through the sera samples of CC patients. Jurkat cells were incubated with $100 \mu \mathrm{l}$ serum of (A) Healthy women or (B) Cancerous women for 48 hrs. Cell were harvested after $48 \mathrm{hrs}$. followed by western blot and densitometry analysis to determine relative extent of JNK activation (ratio between integrated density of phospho-p54/46JNK and total JNK levels). Serum of healthy women induce the p54/46JNK phosphorylation as compared to cancerous patients' sera.

\section{Cervical cancer patient's serum prevents apoptosis} induction in Jurkat cells

Sera samples from HC and CC subjects were added to Jurkat cells and incubated for $48 \mathrm{hrs}$. Results clearly demonstrated that sera from healthy women were able to induce apoptosis ranging from 35.33-65.33\% in Jurkat cells. Whereas highly reduced apoptosis range of 2.50-10.33\% was observed in Jurkat cells after induction with the sera of $\mathrm{CC}$ patients (Fig. $3 \mathrm{~A})$. Overall the average percentage of apoptosis in the $\mathrm{HC}$ with mean $( \pm \mathrm{SE}) 51.29 \pm$ $2.45 \%$ and median $53.50 \%$ whereas corresponding value in $\mathrm{CC}$ patients was significantly lower $(P<0.001)$ with mean $( \pm$ SE) $7.47 \pm 0.57 \%$ and median $7.66 \%$. Apoptosis reduction by $85.4 \%$ was observed in Jurakt cells, when induced with sera from CC patients as compared to sera from HC. In agreements to the previous reports (27); these results indicate that $\mathrm{CC}$ reduces the apoptotic potential of sera to induce cell death in Jurkat cells.

\section{Correlation of sCD95 levels with inhibition of apoptosis in Jurkat cells}

As previous experiments showed the involvement of CD95 pathway in the induction of apoptosis in Jurkat cells. Quantification of sCD95 protein levels in all sera samples was done through ELISA. The sCD95 in HC ranged from 1.00-3.00 ng/ml with mean ( \pm SE) $1.64 \pm$ $0.09 \mathrm{ng} / \mathrm{ml}$ and median $1.66 \mathrm{ng} / \mathrm{ml}$ whereas in CC patients it ranged from $1.45-5.50 \mathrm{ng} / \mathrm{ml}$ with mean $( \pm$ SE) $3.13 \pm 0.21 \mathrm{ng} / \mathrm{ml}$ and median 3.05 $\mathrm{ng} / \mathrm{ml}$. The mean value of sCD95 level was comparatively higher in $\mathrm{CC}$ patients as compared to healthy controls. Comparing the difference between $\mathrm{CC}$ patients and healthy samples for the mean value of sCD95; Student's T-test showed significantly $(\mathrm{p}<0.001)$ higher sCD95 (47.8\%) in CC patients as compared to 
HC (Fig. 3B). Statistical analysis of the experimental data clearly demonstrated a correlation between elevated levels of sCD95 and low apoptosis induction in Jurkat cells, while using CC serum unlike healthy volunteers.
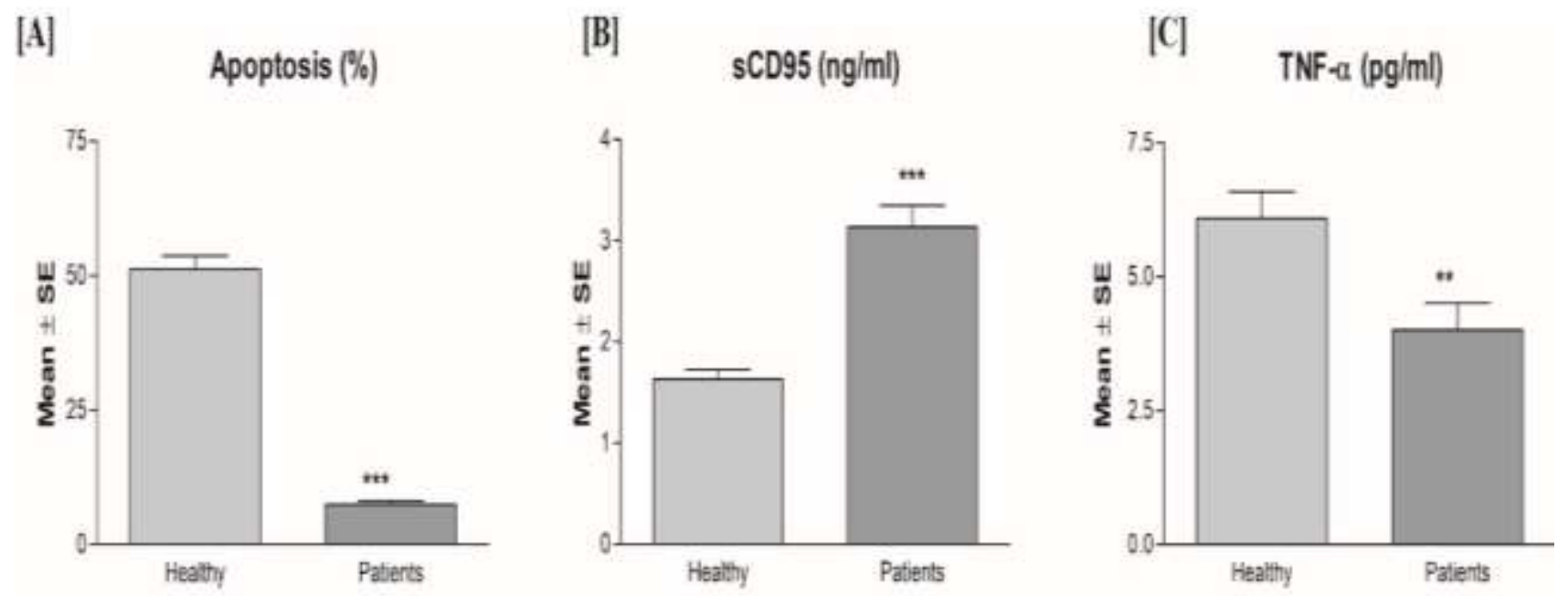

Fig. 3. Differential parameters i.e. apoptosis induction in Jurkat cells as well as sCD95 \& TNF- $\alpha$ concentration in sera samples of HC $(n=20)$ Vs. CC patients $(n=20)$. (A) Induction of apoptosis (\%) in Jurkat cells after incubation with $100 \mu 1$ sera samples of CC patients. (B) Increased levels of sCD95 (Fas receptor) in CC patients' sera as compared to healthy sera samples. (C) Decreased levels of TNF- $\alpha$ in sera samples of CC patients Vs. HC. Concentrations of sCD95 and TNF- $\alpha$ were measured by ELISA in sera obtained from $\mathrm{HC}$ and CC patients separately in triplicate. These results are represented as mean \pm Standard Error of mean. $* * \mathrm{p}<0.01$ or $* * * \mathrm{p}<0.001-$ as compared to HC.

\section{Serum concentration of TNF- $\alpha$ modulates apoptosis in cervical cancer}

TNF- $\alpha$ is a multifunctional cytokine that plays a dual role. While TNF- $\alpha$ could be an endogenous promoter of tumor, as it stimulates cancer cell growth, proliferation, invasion and metastasis as well as tumor angiogenesis. Therefore, the next objective of this study was to assess the level of TNF- $\alpha$ in the serum samples of CC patients as compare to the serum samples of HC. The TNF- $\alpha$ in HC ranged from $2.24-8.50 \mathrm{pg} / \mathrm{ml}$ with mean $( \pm \mathrm{SE}) 6.08 \pm 0.51$ $\mathrm{pg} / \mathrm{ml}$ and median $6.38 \mathrm{pg} / \mathrm{ml}$ whereas in CC patients it ranged from $2.05-9.25 \mathrm{pg} / \mathrm{ml}$ with mean $( \pm$ SE) $4.00 \pm 0.51 \mathrm{pg} / \mathrm{ml}$ and median 3.05 $\mathrm{pg} / \mathrm{ml}$. The mean value of TNF- $\alpha$ level dropped comparatively in $\mathrm{CC}$ patients as compared to HC. Comparing the difference in the mean value of TNF- $\alpha$ between two groups, Student's T-test showed significantly $(\mathrm{p}<0.01)$ lower (34.2\%) level of TNF- $\alpha$ in CC patients as compared to HC (Fig. 3C).

\section{Correlation and best fit regression analysis for all the three parameters}

To find out whether there is any correlation exists between the outcome of these variables (sCD95, TNF- $\alpha$ and apoptosis induction), the inter-correlation analysis was performed. Pearson correlation analysis showed direct (positive) correlation ( $\mathrm{r}=0.21)$ between $\mathrm{SCD} 95$ and TNF- $\alpha$ concentration of sera in $\mathrm{HC}$, whereas a negative (inverse) correlation $(r=-$ 0.22 ) was observed in case of CC patients (Fig. 4A). Further we observed a negative correlation between sCD95 concentration and induced apoptosis in Jurkat cells for both HC ( $\mathrm{r}=-0.07)$ and $\mathrm{CC}$ patients' $(\mathrm{r}=-0.17)$ sera samples (Fig. $4 \mathrm{~B})$. For TNF- $\alpha$ concentration and induced apoptosis; $\mathrm{HC}$ showed a negative correlation $(\mathrm{r}=-0.04)$ in contrast to $\mathrm{CC}$ patients, where a positive correlation ( $\mathrm{r}=0.28$ ) was observed (Fig. 4C). In the group of CC patients, all the three variables showed higher correlation (or trend) as compared to $\mathrm{HC}$ but further studies with the increased number of subjects will correlate these parameters at the significant level. 

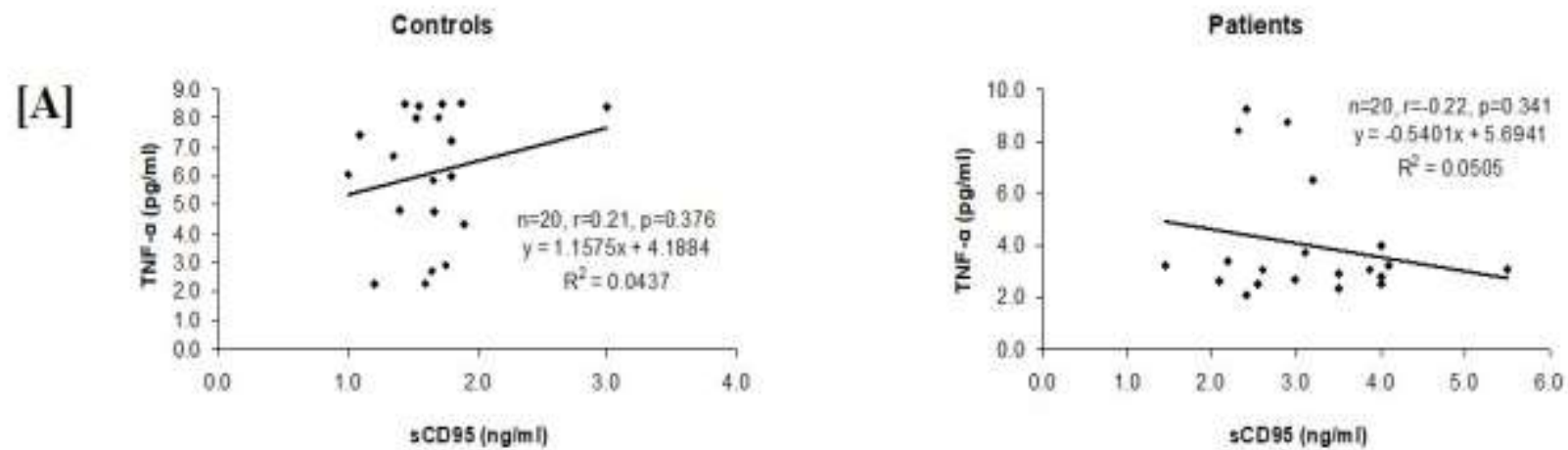

[B]
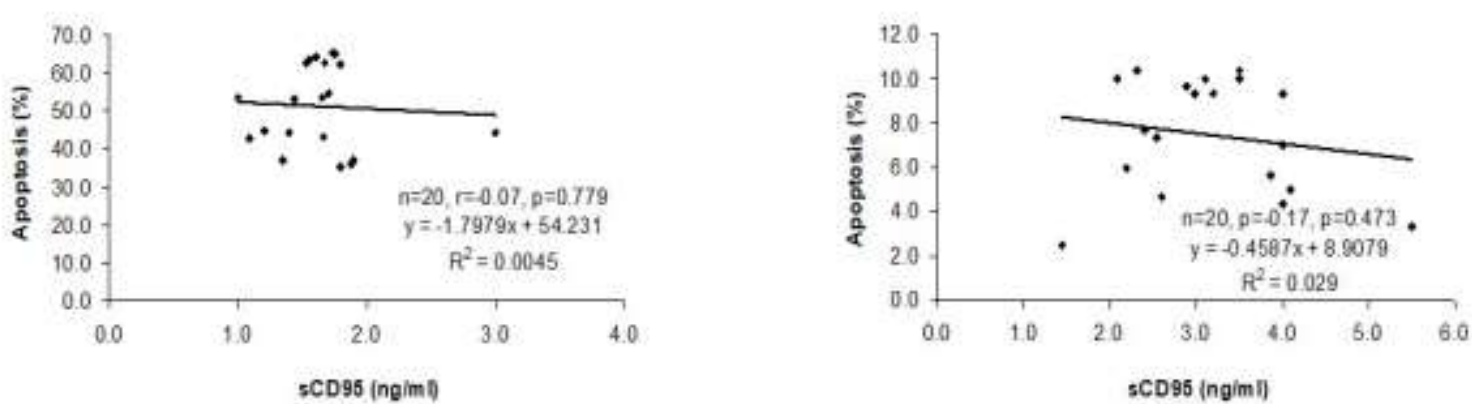

$[\mathrm{C}]$
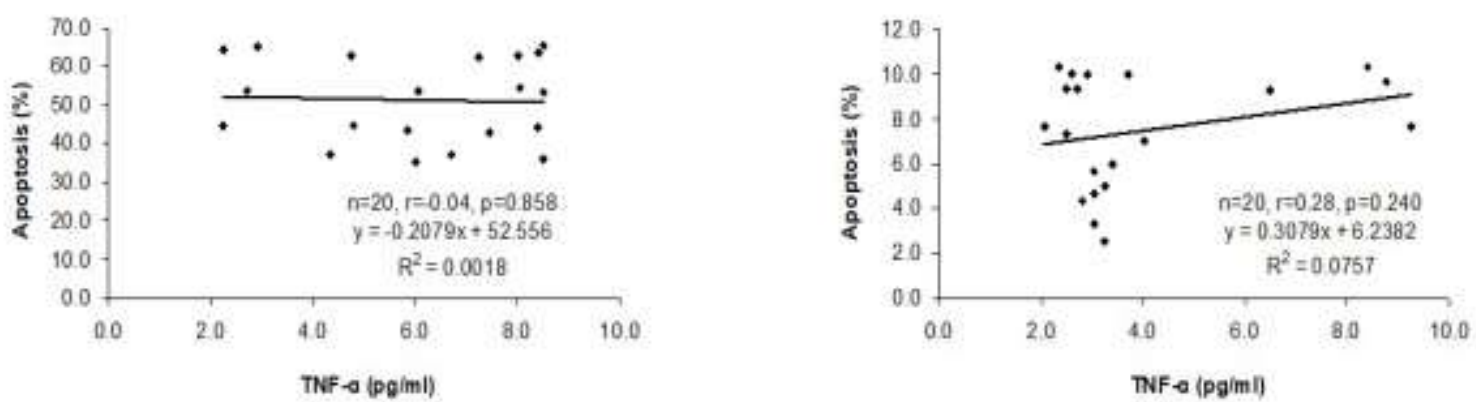

Fig. 4. Correlation and best fit regression analysis for the serum concentration of sCD95 and TNF- $\alpha$ as well as apoptosis induction of Jurkat cells through sera samples of HC Vs. patients. Pearson correlation analysis was done to assess association between the variables. (A) Positive (direct) correlation ( $\mathrm{r}=0.21$ ) was observed between sCD95 and TNF- $\alpha$ concentration of sera in $\mathrm{HC}$, whereas a negative (inverse) correlation $(\mathrm{r}=-0.22)$ was observed in CC patients. (B) Negative correlation was observed between sCD95 concentration and induced apoptosis for both $\mathrm{HC}(\mathrm{r}=-0.07)$ and $\mathrm{CC}$ patients' groups $(\mathrm{r}=-0.17)$. $(\mathrm{C})$ For TNF- $\alpha$ concentration and induced apoptosis, $\mathrm{HC}$ showed a negative correlation $(\mathrm{r}=-0.04)$ in contrast to positive correlation $(\mathrm{r}=0.28)$ of $\mathrm{CC}$ patients.

\section{Sensitivity and specificity for sCD95, TNF- $\alpha$ levels and apoptosis induction to discriminate patients from healthy controls}

To find out diagnostic accuracy (i.e. sensitivity and specificity) for all the three variables (sCD95, TNF- $\alpha$ and apoptosis induction), the Receiver Operative Characteristics (ROC) curve analysis and Area Under Curve (AUC) of each outcome variable was done between healthy controls and patients. The levels of sCD95 \& TNF- $\alpha$ as well as apoptosis induction in Jurkat cells showed significant diagnostic accuracy. Apoptosis induction turned out to be the best parameter in terms of diagnostic accuracy with AUC $=1.000$ ( $\mathrm{p}<0.001)$ for cutoff value of $\leq 10.33 \%$, having $100.00 \%$ sensitivity and specificity (95\% CI: 83.0-100.0) (Fig. 5A). Similarly, for sCD95 cut-off value was observed to be $>1.9 \mathrm{ng} / \mathrm{ml}$ with AUC $=0.942 \quad(\mathrm{p}<0.001) \quad$ having $\quad 95.00 \%$ sensitivity and specificity (95\% CI: 75.1-99.2) (Fig. 5B). Whereas diagnostic accuracy for TNF- $\alpha$ was also assessed which showed AUC $=0.712 \quad(p=0.010)$, with cut-off value $\leq 4.01 \mathrm{pg} / \mathrm{ml}$ having sensitivity and specificity of $80.00 \%$ (95\% CI: 56.3-94.1) (Fig. 5C). 


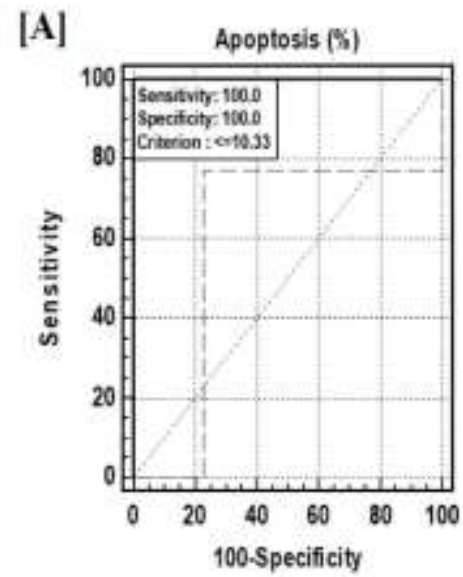

[B]

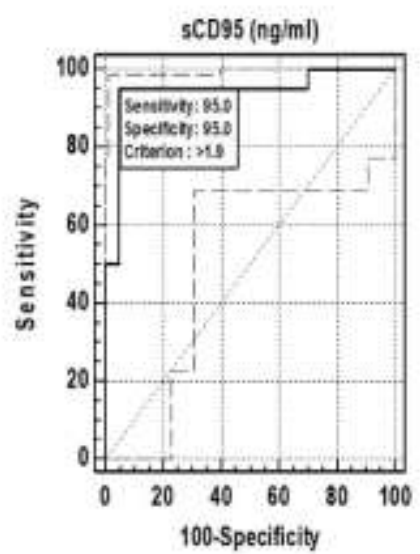

$[\mathrm{C}]$

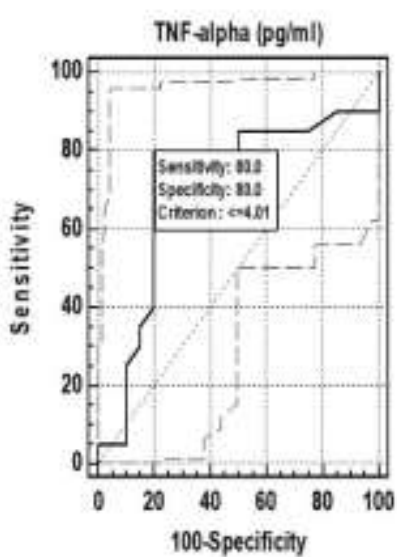

Fig. 5. Receiver operative characteristics (ROC) curve analysis to check diagnostic accuracy for all the three variables and the cut off value to discriminate CC patients from HC. (A) Cut-off for Apoptosis induction (\%) in Jurkat cells was found to be $\leq 10.33 \%$, while its sensitivity and specificity is of $100.0 \%$. (B) Cut-off concentration of sCD95 was found to be $>1.9$ $\mathrm{ng} / \mathrm{ml}$, while its sensitivity and specificity is of $95.0 \%$. (C) Cut-off concentration of TNF- $\alpha$ was found to be $\leq 4.01 \mathrm{pg} / \mathrm{ml}$, while its sensitivity and specificity is of $80.0 \%$.

\section{Discussion}

It is known that CD95-ligand binds to the sCD95, which further protect the cancerous cells from CD95-mediated apoptosis and represents one of the method of evading immune surveillance $(2,28)$. Based on these previous reports, the present study established a cell-based assay, where serum-induced apoptosis in Jurkat cells was directly mediated by the CD95 pathway. At the same time, transformed HEK-293 cells were used as negative control, as these cells are known to be resistant to CD95L and do not die from CD95 apoptosis (Fig. 1).

Interaction of CD95-ligand leads to the activation of death receptor and signal is transmitted to MAP kinase pathway that culminated in apoptosis through JNK1 and p38 phosphorylation. Therefore, in order to check, whether this apoptosis pathway is mediated by JNK1 and p38 phosphorylation and can be induced by FasL-Fas interaction; next objective was to analyze the JNK1 and p38 phosphorylation in Jurkat $\mathrm{T}$ cells during induced apoptosis. Immuno-blotting result of these Jurkat $T$ cells demonstrated that induced apoptosis was mediated by the elevated phosphorylation of JNK as lower phosphorylation of JNK was observed in the un-induced Jurkat $\mathrm{T}$ cells, which were incubated with the CC patients' sera samples. Similarly, increased phosphorylation of p38 was also detected (data not shown); hence it can be depicted that serum of healthy volunteer induces the apoptosis through JNK1 and p38 phosphorylation (Fig. 2).

Induced apoptosis of Jurkat cells reduced significantly, when cells were supplemented with sera samples of $\mathrm{CC}$ patients, whereas supplementation with healthy sera samples resulted in higher apoptotic indices (Fig. 3A). Based upon these observations, which suggested a crucial role of CD95 in this mechanism; the next objective was to check the sCD95 levels in the serum of all the CC patients. This experiment showed elevated levels of sCD95 in the sera of CC patients as compared to healthy control subjects (Fig. 3B). Reports about the other malignancies also authenticated this data as the elevated levels of sCD95 have been observed in patients' sera samples of ovarian, gall bladder, lung, melanoma, head and neck (29), renal carcinomas (30) as well as autoimmune rheumatic diseases (3). Sera levels of sCD95 has also been well correlated with the survival rate of the $\mathrm{CC}$ patients as the lower sCD95 
levels $(<1.5 \mathrm{ng} / \mathrm{ml})$ show better survival rate (27), when compared to the patients having higher sCD95 levels ( $>1.5 \mathrm{ng} / \mathrm{ml})$. Present data is in absolute agreement with these reports, equally present study also found very low levels of sCD95 in the sera samples of HC.

The next segment of the study was TNF- $\alpha$; in order to understand the role of TNF- $\alpha$ in CC, its concentration was assessed in the sera samples. According to the previous studies, TNF- $\alpha$ has been reported in dual roles; where it regulates the proliferation of cell through activation of $\mathrm{NF} \kappa \mathrm{B}$ and induce the apoptosis in cancer killing cell $(31,32)$. TNF- $\alpha$ tends to affect most of the body's organs, and cytokine performs a range of functions, many of which are still unexplored. In the present study, lower levels of TNF- $\alpha$ were observed in the sera samples of the $\mathrm{CC}$ patients as compared to the HC (Fig. 3C). Higher concentration of TNF- $\alpha$ in healthy women may regulate discrete signaling pathways associated with nuclear factor $\kappa \mathrm{B}(\mathrm{NF}-\mathrm{\kappa B})$ and $\mathrm{c}$-Jun N-terminal kinase (JNK). NF- $\mathrm{KB}$ is a foremost antiapoptotic cell survival signal while sustained JNK activation leads to cell death. In fact, the crosstalk between NF- $\mathrm{KB}$ and JNK is involved in determining the cellular outcomes in response to $\mathrm{TNF}-\alpha(6)$.

Correlation and best fit regression analysis between sCD95, TNF- $\alpha$ and apoptosis induction (in Jurkat cells) for CC patients and $\mathrm{HC}$ was done through Pearson correlation analysis to assess association between the variables. In CC patients, it was observed that the lower levels of TNF- $\alpha$ were strongly associated with the reduction in induced apoptosis as compare to the $\mathrm{HC}$; whereas association between sCD95 \& TNF- $\alpha$ for CC patients turned out to be negatively correlated, which showed positive correlation for the HC.

\section{References}

1. Danial NN, Korsmeyer SJ. Cell Death: Critical Control Points. Cell. 2004;116(2):205-19.

2. Walczak H, Krammer PH. The CD95 (APO$1 / \mathrm{Fas}$ ) and the TRAIL (APO-2L) apoptosis systems. Exp Cell Res. 2000 Apr;256(1):58-66.
At the same time, correlation between sCD95 \& induced apoptosis was found to be negative for both $\mathrm{CC}$ patients and $\mathrm{HC}$, but the correlation coefficient was slightly higher for the $\mathrm{CC}$ patients as compare to the HC (Fig. 4).

Further, to assess the diagnostic accuracy of these variables (sCD95, TNF- $\alpha$ and induced apoptosis) through their sensitivity and specificity, ROC curve and AUC analysis was done. Highly significant accuracy was observed for the apoptosis induction; hence this parameter can be widely recommended to assess the risk of the disease. Increased sera levels of sCD95 in patients, was also found to be significant, whereas decreased sera levels of TNF- $\alpha$ in CC patients showed slightly lower diagnostic accuracy (Fig. 5). But at the same time, it is important to consider all the three parameters together, as the apoptotic pathways are usually dependent on both the other parameters.

It is evident from the present study that the distinct sera levels of sCD95 \& TNF- $\alpha$ in CC patients showed an anti-apoptotic effect, which may create a micro-environment to evade the anti-tumor immune response. Hence, it has been suggested that these parameters can be explored for the risk assessment of CC. However, more elaborative studies can be done with larger sample size for the further deployment in the clinical scenario.

\section{Acknowledgements}

Sophisticated Analytical Instrumental Facility of the CSIR-Central Drug Research Institute Lucknow is thankfully acknowledged. This work was supported by Council of Scientific and Industrial Research (CSIR), New Delhi, Government of India.

The authors have no conflicts of interest to declare.

3. Jodo S, Kobayashi S, Kayagaki N, Ogura N, Feng Y, Amasaki Y, et al. Serum levels of soluble Fas/APO-1 (CD95) and its molecular structure in patients with systemic lupus erythematosus (SLE) and other autoimmune diseases. Clin Exp Immunol. 1997;107(1):89-95. 
4. Townson JL, Naumov GN, Chambers AF. The role of apoptosis in tumor progression and metastasis. Curr Mol Med. 2003 Nov;3(7):631-42. 5. Igney FH, Krammer PH. Immune escape of tumors: apoptosis resistance and tumor counterattack. J Leukoc Biol. 2002;71(6):907-20. 6. Liu ZG, Hsu H, Goeddel D V., Karin M. Dissection of TNF receptor 1 effector functions: JNK activation is not linked to apoptosis while NF- $\mathrm{BB}$ activation prevents cell death. Cell. 1996;87(3):565-76.

7. Nagata S. Fas ligand-induced apoptosis. Annu Rev Genet. 1999;33:29-55.

8. Chang HY, Yang X, Baltimore D. Dissecting Fas signaling with an altered-specificity deathdomain mutant: Requirement of FADD binding for apoptosis but not Jun N-terminal kinase activation. Proc Natl Acad Sci USA. 1999;96(4):1252-6.

9. Kondera-Anasz Z, Mielczarek-Palacz A, Sikora J. Soluble Fas receptor and soluble Fas ligand in the serum of women with uterine tumors. Apoptosis. 2005;10(5):1143-9.

10. Suda T, Nagata S. Purification and characterization of the fas-ligand that induces apoptosis. J Exp Med. 1994;179(3):873-9.

11. Oehm A, Behrmann I, Falk W, Pawlita M, Maier G, Klas C, et al. Purification and molecular cloning of the APO-1 cell surface antigen, a member of the tumor necrosis factor/nerve growth factor receptor superfamily. Sequence identity with the Fas antigen. J Biol Chem. 1992;267(15):10709-15.

12. Itoh $\mathrm{N}$, Yonehara S, Ishii A, Yonehara M, Mizushima S, Sameshima M, et al. The polypeptide encoded by the cDNA for human cell surface antigen Fas can mediate apoptosis. Cell. 1991;66(2):233-43.

13. Alderson MR, Tough TW, Davis-Smith T, Braddy S, Falk B, Schooley KA, et al. Fas ligand mediates activation-induced cell death in human T lymphocytes. J Exp Med. 1995;181(1):71-7.

14. Mizutani Y, Yoshida O, Bonavida B. Prognostic significance of soluble Fas in the serum of patients with bladder cancer. J Urol. 1998;160(2):571-6.

15. Ueno T, Toi M, Tominaga T. Circulating soluble Fas concentration in breast cancer patients. Clin Cancer Res. 1999;5(11):3529-33.
16. Hatai T, Matsuzawa A, Inoshita S, Mochida Y, Kuroda T, Sakamaki K, et al. Execution of apoptosis signal-regulating kinase 1 (ASK1)induced apoptosis by the mitochondria-dependent caspase activation. J Biol Chem. 2000;275(34):26576-81.

17. Wentzensen N, Von Knebel Doeberitz M. Biomarkers in cervical cancer screening. Dis Markers. 2007;23(4):315-30.

18. Lettini AA, Guidoboni M, Fonsatti E, Anzalone L, Cortini E, Maio M. Epigenetic remodelling of DNA in cancer. Histol Histopathol. 2007;22(10-12):1413-24.

19. Syrjänen KJ. Spontaneous evolution of intraepithelial lesions according to the grade and type of the implicated human papillomavirus (HPV). Eur J Obstet Gynecol Reprod Biol. 1996;65(1):45-53.

20. Kanamoto T, Mota M, Takeda K, Rubin LL, Miyazono K, Ichijo H, et al. Role of apoptosis signal-regulating kinase in regulation of the c-Jun N-terminal kinase pathway and apoptosis in sympathetic neurons. Mol Cell Biol. 2000;20(1):196-204.

21. Zur Hausen H. Papillomaviruses and cancer: From basic studies to clinical application. Nat Rev Cancer. 2002;2(5):342-50.

22. Bosch FX, De Sanjosé S. The epidemiology of human papillomavirus infection and cervical cancer. Dis Markers. 2007;23(4):213-27.

23. Walboomers J, Jacobs M, Manos M, Bosch F, Kummer J, Shah K, et al. Human Papillomavirus Is a Necessary Cause. J Pathol. 1999; 189:12-9.

24. Kischkel FC, Lawrence DA, Tinel A, LeBlanc H, Virmani A, Schow P, et al. Death receptor recruitment of endogenous caspase-10 and apoptosis initiation in the absence of caspase8. J Biol Chem. 2001;276(49):46639-46.

25. Lavrik I, Golks A, Krammer PH. Death receptor signaling. J Cell Sci. 2005;118(2):265-7. 26. Smith CA, Farrah T, Goodwin RG. The TNF receptor superfamily of cellular and viral proteins: activation, costimulation, and death. Cell. 1994;76(6):959-62.

27. Aguilar-Lemarroy A, Romero-Ramos JE, Olimon-Andalon V, Hernandez-Flores G, LermaDiaz JM, Ortiz-Lazareno PC, et al. Apoptosis induction in Jurkat cells and sCD95 levels in 
women's sera are related with the risk of developing cervical cancer. BMC Cancer. 2008;8:1-12.

28. Vejda S, Posovszky C, Zelzer S, Peter B, Bayer E, Gelbmann D, et al. Plasma from cancer patients featuring a characteristic protein composition mediates protection against apoptosis. Mol Cell Proteomics. 2002;1(5):38793.

29. Midis GP, Shen Y, Owen-Schaub LB. Elevated soluble Fas (sFas) levels in nonhematopoietic human malignancy. Cancer Res. 1996;56(17):3870-4.
30. Kimura M, Tomita $Y$, Imai $T$, Saito $T$, Katagiri A, Tanikawa T, et al. Significance of serum-soluble CD95 (Fas/APO-1) on prognosis in renal cell cancer patients. $\mathrm{Br} \mathrm{J}$ Cancer. 1999;80(10):1648-51.

31. Yang J, Lin Y, Guo Z, Cheng J, Huang J, Deng $\mathrm{L}$, et al. The essential role of MEKK3 in TNF-induced NF- $\mathrm{\kappa B}$ activation. Nat Immunol. 2001;2(7):620-4. A

32. Devin A, Cook A, Lin Y, Rodriguez Y, Kelliher M, Liu Z. The distinct roles of TRAF2 and RIP in IKK activation by TNF-R1: TRAF2 recruits IKK to TNF-R1 while RIP mediates IKK activation. Immunity. 2000;12(4):419-29. 